\title{
The transpulmonary pressure gradient for the diagnosis of pulmonary vascular
} disease

\author{
Robert Naeije*, Jean-Luc Vachiery ${ }^{\#}$, Patrick Yerly ${ }^{\#}$ and Rebecca Vanderpool*
}

ABSTRACT: The transpulmonary pressure gradient (TPG), defined by the difference between mean pulmonary arterial pressure $(P p a)$ and left atrial pressure ( $P$ la; commonly estimated by pulmonary capillary wedge pressure: $P$ pcw) has been recommended for the detection of intrinsic pulmonary vascular disease in left-heart conditions associated with increased pulmonary venous pressure. In these patients, a TPG of $>12 \mathrm{mmHg}$ would result in a diagnosis of "out of proportion" pulmonary hypertension. This value is arbitrary, because the gradient is sensitive to changes in cardiac output and both recruitment and distension of the pulmonary vessels, which decrease the upstream transmission of Pla. Furthermore, pulmonary blood flow is pulsatile, with systolic Ppa and mean Ppa determined by stroke volume and arterial compliance. It may, therefore, be preferable to rely on a gradient between diastolic $P$ pa and $P$ pcw. The measurement of a diastolic $P$ pa/Ppcw gradient (DPG) combined with systemic blood pressure and cardiac output allows for a step-by-step differential diagnosis between pulmonary vascular disease, high output or high leftheart filling pressure state, and sepsis. The DPG is superior to the TPG for the diagnosis of "out of proportion" pulmonary hypertension.

KEYWORDS: Heart failure, pulmonary capillary wedge pressure, pulmonary circulation, pulmonary hypertension, pulmonary vascular compliance, pulmonary vascular resistance

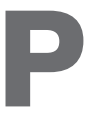

ulmonary hypertension is defined by a mean pulmonary arterial pressure $(P$ pa $)$ $\geqslant 25 \mathrm{mmHg}$ at rest $[1,2]$. The diagnosis of pulmonary vascular disease relies on: invasive measurements of mean $P$ pa $\geqslant 25 \mathrm{mmHg}$; a pulmonary capillary wedge pressure $(P \mathrm{pcw}) ; \leqslant 15 \mathrm{mmHg}$; a pulmonary vascular resistance $(\mathrm{PVR}) \geqslant 3$ Wood units; and a transpulmonary pressure gradient $(\mathrm{TPG}) \geqslant 12 \mathrm{mmHg}[1,2]$. The TPG is the difference between mean $P$ pa and left atrial pressure $(P l a)$. Pla is usually estimated by $P$ pcw. The TPG is thought to be particularly useful to diagnose "out of proportion pulmonary hypertension" in patients with left heart failure or mitral stenosis [1]. "Out of proportion" infers that mean $P$ pa is higher than expected from an upstream transmission of $\mathrm{Pla}$, because of increased tone and/or structural changes. However, a TPGderived diagnosis of "out of proportion" pulmonary hypertension may not always agree with clinical context.

The degree of pulmonary hypertension that is the passive consequence of increased $\mathrm{Pla}$ due to advanced left heart failure can be observed in cardiac transplantation. In patients with purely passive pulmonary hypertension, the mean $P$ pa would decrease along with decreased $P$ la, while in those with "out of proportion" pulmonary hypertension the mean Ppa would remain unchanged, or decrease proportionally less than Pla. This was examined in 20 previously reported patients with pre- and post-operative haemodynamic measurements [3]. Before transplantation the mean \pm SE was: $43 \pm 2 \mathrm{mmHg}$ for $P$ pa; $29 \pm 2 \mathrm{mmHg}$ for $P$ pcw, $604 \pm 60 \mathrm{dyn} \cdot \mathrm{s}^{-1} \cdot \mathrm{cm}^{-5} \cdot \mathrm{m}^{-2}$ for PVRi; $14 \pm 1 \mathrm{mmHg}$ for TPG. After transplantation: the mean \pm SE was $25 \pm 2 \mathrm{mmHg}$ for $P$ pa; $12 \pm 1 \mathrm{mmHg}$ for $P$ pcw,
For editorial comments see page 7.

\section{AFFILIATIONS}

*Dept of Physiology, Faculty of Medicine, Free University Brussels, and

\#Dept of Cardiology, Erasme University Hospital, Free University Brussels, Brussels, Belgium.

\section{CORRESPONDENCE}

$R$ Naeije

Dept of Physiology

Faculty of Medicine

Free University of Brussels

808 Lennik Road

1070-Brussels

Belgium

E-mail: rnaeije@ulb.ac.be

Received:

Accepted after revision:

July 262012

First published online:

Aug 302012
May 102012 
$452 \pm 50 \mathrm{dyn} \cdot \mathrm{s}^{-1} \cdot \mathrm{cm}^{-5} \cdot \mathrm{m}^{-2}$ for PVRi; and $12 \pm 1 \mathrm{mmHg}$ for TPG. In this series of patients, transplantation was followed by a proportional decrease in mean $P$ pa and $P$ pcw (by, on average, $18 \mathrm{mmHg}$ and $17 \mathrm{mmHg}$, respectively) suggestive of purely passive pulmonary hypertension. Yet the initial TPG was, on average, $>12 \mathrm{mmHg}$, with a range of values from $6-20 \mathrm{mmHg}$. Since transplantation did not always normalise mean $P$ pa, the data taken from 12 out of the 20 patients in whom mean $P$ pa decreased to below the value of $25 \mathrm{mmHg}$ were re-examined. The preoperative TPG was $>12 \mathrm{mmHg}$ (range $12-20 \mathrm{mmHg}$ ) in six of the patients. Thus, in this limited series of patients, a TPG $>12 \mathrm{mmHg}$ did not predict "out of proportion" pulmonary hypertension better than flipping a coin. How is this possible?

\section{HOW LEFT ATRIAL PRESSURE AFFECTS MEAN PULMONARY ARTERIAL PRESSURE}

The normal relationship between mean $P$ pa and $P$ la is described by the PVR equation rearranged as: mean $P$ pa $=P V R \times Q+P l a$, where $\mathrm{Q}$ is pulmonary blood flow. The inherent assumptions of the PVR equation are that the TPGT-Q relationship is linear, crosses a zero pressure/zero flow value, and is, therefore, independent of the absolute value of Pla. Many studies have shown that the TPG-Q relationship may be reasonably well described by a linear approximation over a limited range of physiological flows, but that its extrapolated pressure intercept is most often positive, and that the slope of the mean $\mathrm{Ppa}-\mathrm{Pla}$ relationship is less than the unity [4].

It has long been known that PVR decreases with increases in Q or Pla. This has been initially explained by a pulmonary circulation model of parallel collapsible vessels with a distribution of closing pressures $\left(P_{c}\right)$ [5]. In each of these vessels, flow is determined by a pressure gradient between $P_{p a}$ and $P_{\mathrm{c}}$ whenever there is a $P_{\mathrm{c}}>P_{\text {la }}$. A $P$ la lower than $P_{\mathrm{c}}$ is irrelevant to flow; like the height of a waterfall. Pla then becomes an apparent outflow pressure and $P_{\mathrm{c}}$ the effective outflow pressure of the pulmonary circulation, while the PVR equation remains valid provided $P$ la is replaced by $P_{\mathrm{c}}$. When $P$ la is higher than $P_{\mathrm{c}}$, the vessel opens and the driving pressure for flow becomes $P$ pa minus $P$ la, the effective outflow pressure of the pulmonary circulation is Pla and the usual PVR equation can be used.

A $P_{\mathrm{c}}$ higher than $P_{\text {la }}$ is typically observed in West's zones I and II in the upper parts of normal vertical lungs [6]. Diseases associated with increased pulmonary vessel tone and/or alveolar pressure $(P A)$ are associated with an increase in $P_{c}$. In these patients, mean $P$ pa becomes less sensitive or even insensitive to changes in flow or Pla, and calculated PVR rapidly decreases with increased cardiac output [7]. The presence of a closing pressure in the pulmonary circulation can be identified by a gradient between $P$ la and the extrapolated pressure intercept of the linear adjustment of a multiple mean $P$ pa-Q coordinates measured at constant Pla. Further proof is brought about by the demonstration of a functional dissociation between $P_{c}$ and $P$ la on mean $P$ pa $-P$ la relationships in experimental preparations in which flow is kept constant; this is discussed further by NAEIJE [3]. A typical experiment in an intact animal preparation of oleic acid lung injury, as a model of the acute respiratory distress syndrome, is shown in figure 1 [8]. In this animal, the extrapolated intercept of linear mean $P$ pa-Q plots revealed a $P_{\mathrm{c}}$ higher than $P$ la, and $P$ la had to be increased above that value to be transmitted upstream to mean $P$ pa.

The "waterfall model"" of the pulmonary circulation does not take into account the natural distensibility of the pulmonary vessels. A sufficient number of mean $P$ pa-Q coordinates, $>4-5$, show a slight curvilinearity, which is ignored by linear adjustment procedures. This curvilinearity is explained by the fact that a high flow distends pulmonary resistive vessels, and is

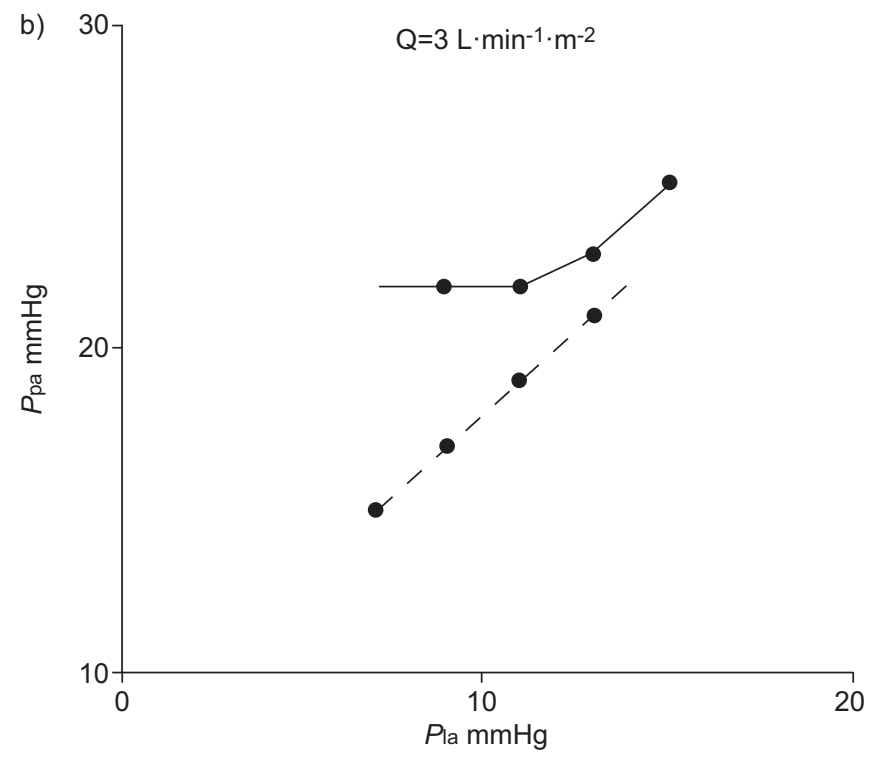

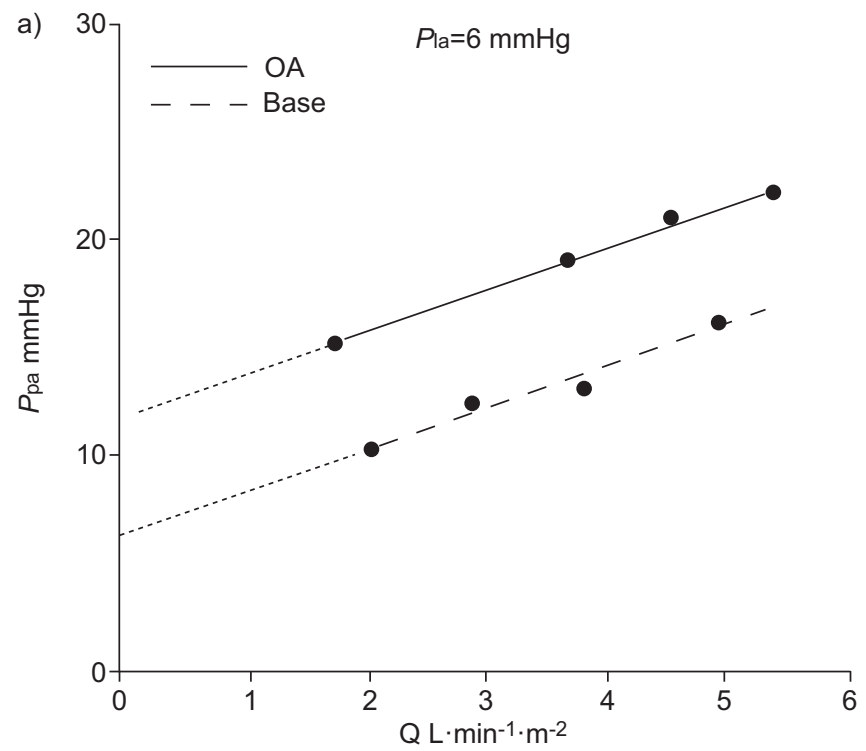

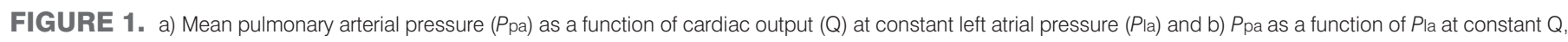

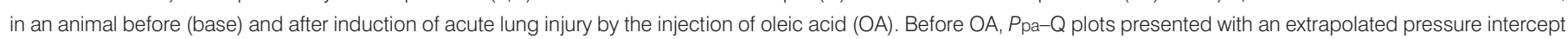

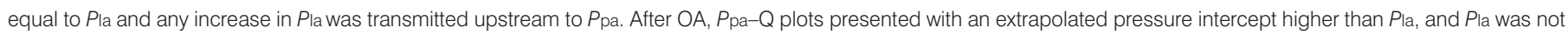

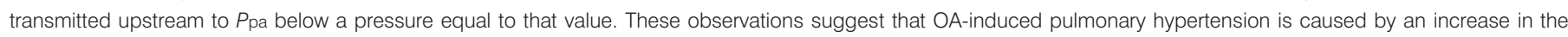
closing pressure of the pulmonary circulation. Data from [8]. 
an important cause of decreased slope of mean $P$ pa-Q relationships, or PVR along with increasing flow. High flow linear adjustments of multipoint mean $P$ pa-Q relationships are, therefore, associated with spurious increase in $P_{c}$ estimated from extrapolated pressure intercepts [9].

This difficulty is overcome by a mathematical model of the pulmonary circulation fitting multipoint mean $P$ pa-Q plots by an equation relating mean $P$ pa, $P$ la, $Q$, total PVR at rest (R0) and a distensibility coefficient $(\alpha)[9]$ :

$$
\text { mean } P_{\mathrm{pa}}=\left(\left[\left(1+\alpha \mathrm{Pla}^{5}+5 \alpha \mathrm{R} 0(\mathrm{Q})\right]^{1 / 5}-1\right) / \alpha\right.
$$

The normal value for $\alpha$ measured on in vitro mounted pulmonary resistive vessels is $2 \%$ change in diameter per $\mathrm{mmHg}$ change in pressure, and is remarkably constant in a wide spectrum of animal species [10]. It is interesting that the same distensibility $\alpha$-value of $2 \%$ per $\mathrm{mmHg}$ has been recovered by the application of the distensibility model equation to either invasive [10] or noninvasive [11-13] measurements of pulmonary vascular pressures and flows. The distensibility factor $\alpha$ is higher in young healthy females when compared with males [12], and decreases with aging [12] or with chronic hypoxic exposure [13].

The distensibility equation allows for the modelling of the effects of increased Q or Pla on mean $P$ pa at various levels of vascular distensibility and PVR. The results are shown in figures 2 and 3. It is apparent that an increase in $\alpha$ decreases mean $P$ pa or TPG at any given level of flow (figs 2 and 3) and also decreases the TPG along with an increase in $P$ pcw (fig. 3) In other words, an increase in $\mathrm{Q}$ and/or Pla may falsely decrease the TPG that would ordinarily be increased because of the pulmonary vasoconstriction or remodelling.

Distensible models provide a satisfactory explanation for all possible normal or pathological pulmonary vascular pressureflow relationships in fully recruited lungs. Pulmonary vascular

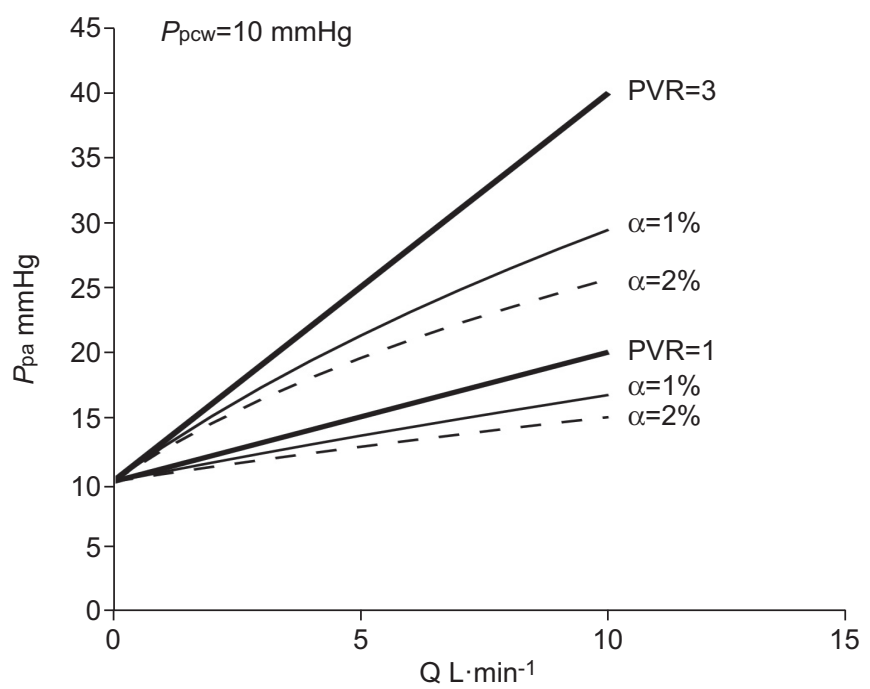

FIGURE 2. Mean pulmonary arterial pressure $(P \mathrm{pa})$ as a function of cardiac output (Q) at two different levels of pulmonary vascular resistance (PVR). Increasing distensibility $(\alpha)$ decreases $P$ pa at any given level of $Q$. Thus pulmonary vascular distensibility results in a $P$ pa that is less than the one predicted by a linear model, which is the PVR equation. de-recruitment has to be taken into account in low cardiac output or high alveolar pressure states. The slope of the mean $P$ pa-Pla relationship decreases with pulmonary vascular distension, but may increase in de-recruited lungs. In that case, an increased TPG may falsely suggest pulmonary vasoconstriction or remodelling.

There is thus no good rationale for a stable cut-off value of $12 \mathrm{mmHg}$ for the TPG as the measurement is sensitive to Pladependent changes in pulmonary vascular recruitment and distension

\section{HOW LEFT ATRIAL PRESSURE AFFECTS PULSATILE PULMONARY ARTERIAL PRESSURE}

The above considerations do not take into account the natural pulsatility of the pulmonary circulation. Pulmonary flow reaches a maximum during systole, and is inappreciable at the end of diastole. Accordingly, in a normal pulmonary circulation, diastolic $P$ pa is approximately equal to Pla [14]. It has been assumed, after taking into account errors for measurements of $\pm 1-2 \mathrm{mmHg}, 5 \mathrm{mmHg}$ would be a reasonable upper limit for normal diastolic $P$ pa $/ P$ pcw gradient. This is indeed what was established on the basis of invasive hemodynamic measurements in 44 healthy volunteers aged $17-83$ yrs, at rest and at various levels of exercise in either recumbent and sitting positions associated with $P$ pcw values up to $34 \mathrm{mmHg}$ and cardiac outputs of up to $25-30 \mathrm{~L} \cdot \mathrm{min}^{-1}[14]$. Systolic $P$ pa and mean $P$ pa, at any given diastolic $P$ pa, increase in a fixed proportion depending on stroke volume (SV) and pulmonary arterial compliance ( $\mathrm{Cpa}$, which decreases along with increased $P$ la. Therefore systolic $P$ pa and mean $P$ pa can be predicted from diastolic Ppa using the following equations [14]:

$$
\begin{aligned}
& \text { systolic } P \text { pa }=1.41+1.61 \text { diastolic } P \text { pa }+0.09 \mathrm{SV} \\
& \text { mean } P_{\text {pa }}=-1.33+1.34 \text { diastolic } P \text { pa }+0.05 \mathrm{SV}
\end{aligned}
$$

Predicted mean $P$ pa and systolic $P$ pa as a function of $P$ pcw at various SVs are illustrated in figures 4-6.

It now appears that the TPG may remain unchanged if diastolic $P$ pa increases less than $P$ pcw, but increases if diastolic $P$ pa increases by an equal amount or more than $P$ pcw. Alternatively, the diastolic $P$ pa $/ P$ pcw gradient (DPG) decreases or remains unchanged as long as the upstream transmission of $P$ pcw remains equal or less than the unity, which is expected in the absence of pulmonary vasoconstriction or remodelling.

The tight correlation between systolic, mean, and diastolic Ppa was recently rediscovered, with similar prediction equations that interestingly remained valid in pulmonary hypertension of various severities and aetiologies [15-17]. This is explained by the monotonous response of the pulmonary circulation to insults, leading to proportional inverse changes in Cpa and PVR with an unaltered time constant $C$ pa $\times$ PVR 0.6-0.7 s [18, 19]. There may be one noticeable exception: pulmonary hypertension on passive upstream transmission of increased Pla in left heart conditions. In these patients $C$ pa decreases proportionally more than the increase in PVR, because increased Pla is a cause of both pulmonary arterial stiffening and decreased PVR. Accordingly, the time constant in pulmonary hypertension on left heart conditions is shorter than in other types of pulmonary hypertension [20]. This spuriously increases the TPG, but does not affect DPG. 

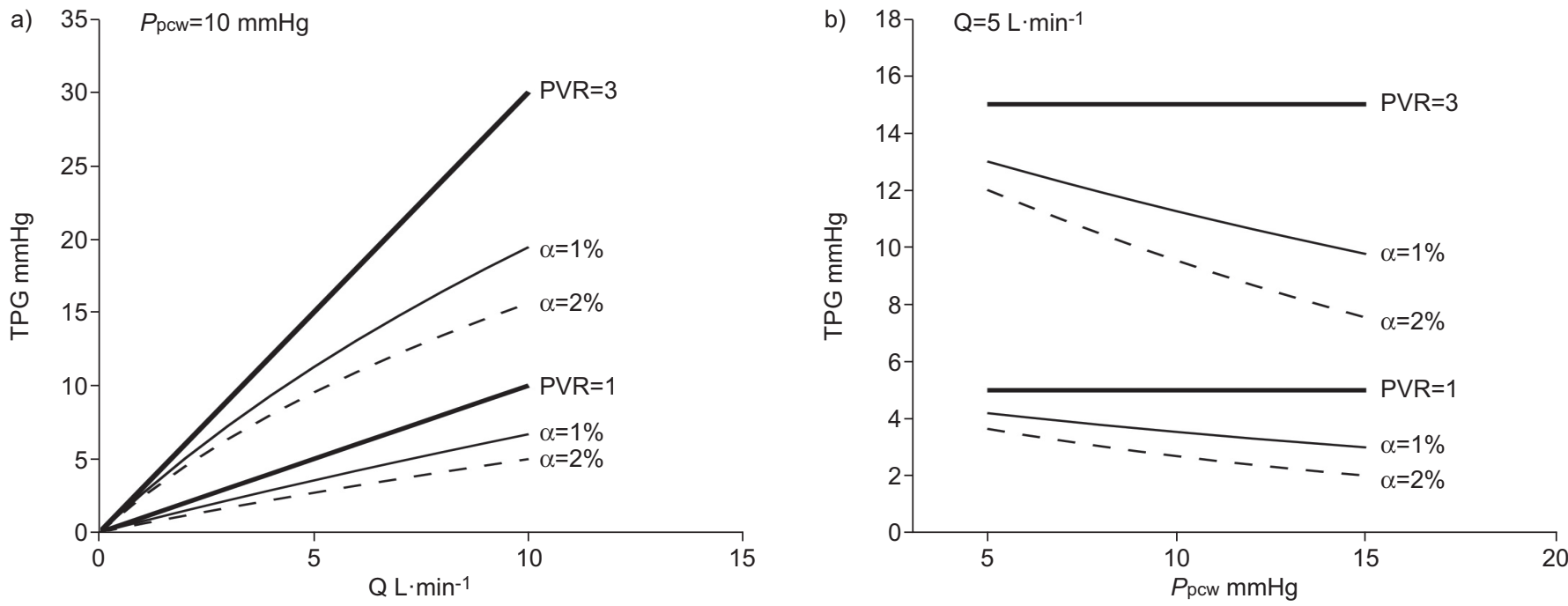

FIGURE 3. In the linear model, the transpulmonary pressure gradient (TPG) is only a function of flow rate (Q) as shown in a), and is not affected by pulmonary capillary wedge pressure $(\mathrm{Ppcw})$, as shown in b), whatever the pulmonary vascular resistance. An increase in pulmonary vascular distensibility decreases the TPG as a function of $Q$ as well as of $P$ pcw.

It must be reminded that $C$ pa should not be confused with the distensibility coefficient $\alpha$. Cpa is a global calculation, influenced by proximal pulmonary arterial elasticity, while distensibility coefficient $\alpha$ strictly corresponds to the distensibility of small peripheral pulmonary resistive vessels.

Thus the disproportionate decrease in $C_{p a}$ in the presence of increased Pla may be a cause of increased TPG without any coexistent pulmonary vasoconstriction or remodelling. How this may cancel out the decrease in TPG, related to pulmonary vascular recruitment and distension, is unpredictable.

Clinicians, understandably, like to have cut-off values for decision making purposes that are based on haemodynamic measurements. We regret to have to tell them that a TPG of $12 \mathrm{mmHg}$ cannot be used for that purpose. The DPG may be

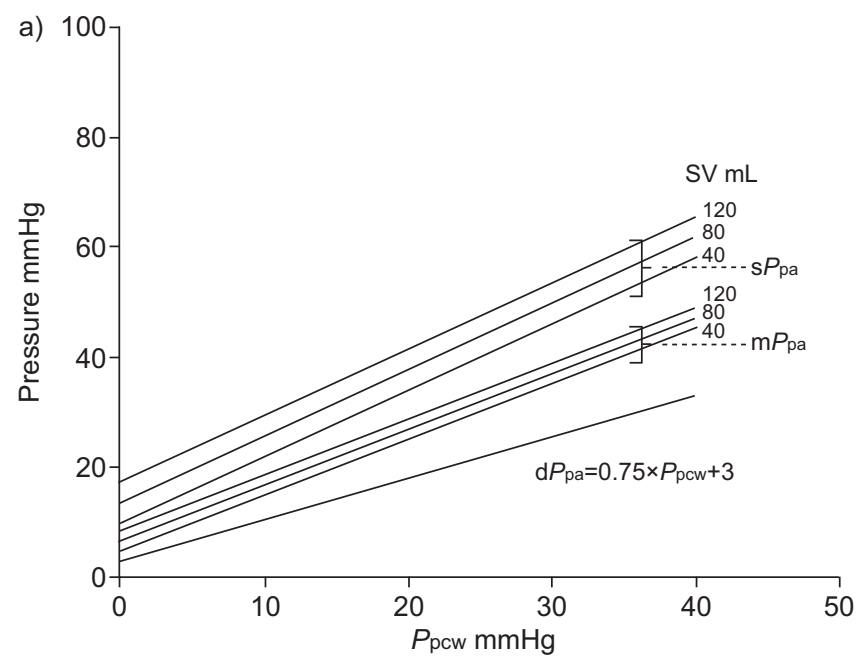

preferable because this gradient is less sensitive to changes in Cpa, SV, and absolute values of Pla.

\section{THE DPG FOR THE DIFFERENTIAL DIAGNOSIS OF PULMONARY HYPERTENSION}

The DPG used to be implemented in the assessment of cardiac versus pulmonary causes of acute respiratory failure in critically ill patients [21]. Here we propose an adaptation of this DPG-derived decision tree to make it more generally applicable (fig. 7). The previous decision tree rested on a cutoff value for $P$ pcw of $10 \mathrm{mmHg}$. A $P$ pcw of $12 \mathrm{mmHg}$, or a direct measure of left ventricular end-diastolic pressure of $15 \mathrm{mmHg}$, would seem a more reasonable cut-off value as extreme upper limits of normal; as used in diagnostic algorithms of diastolic heart failure [22]. The next step is a

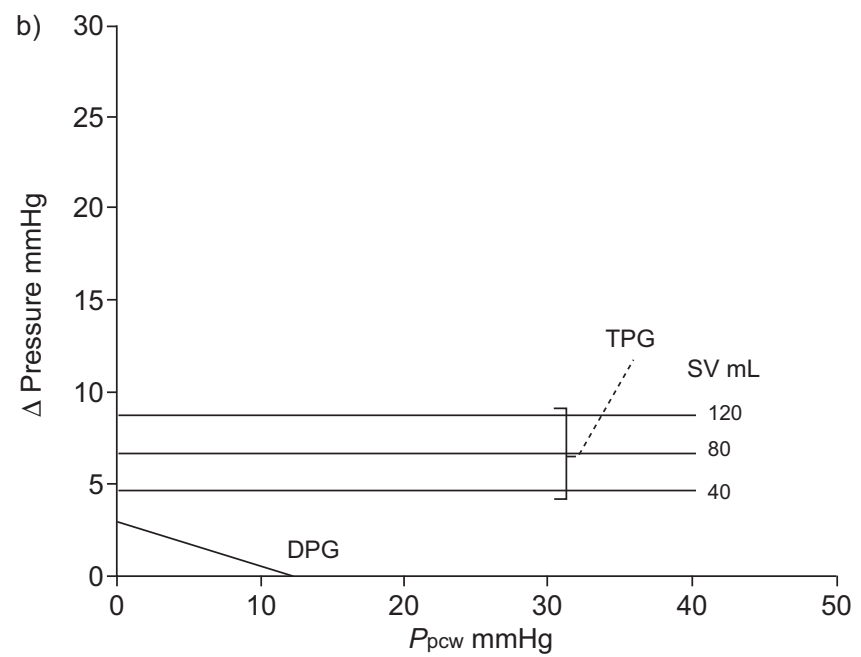

FIGURE 4. Effects of pulmonary capillary wedge pressure ( $P$ pcw) and stroke volume $(\mathrm{SV})$ on systolic (s), diastolic (d) and mean (m) pulmonary arterial pressures (Ppa). If only a fraction of $P$ pcw is transmitted to diastolic $P$ pa, the transpulmonary pressure gradient (TPG) is not a function of $P$ pcw but increases proportionally with SV. The diastolic $P$ pa/Ppcw gradient (DPG) decreases with increased $P$ pcw, independently of SV. The equations from [14] were used to model the response of the vasculature. 

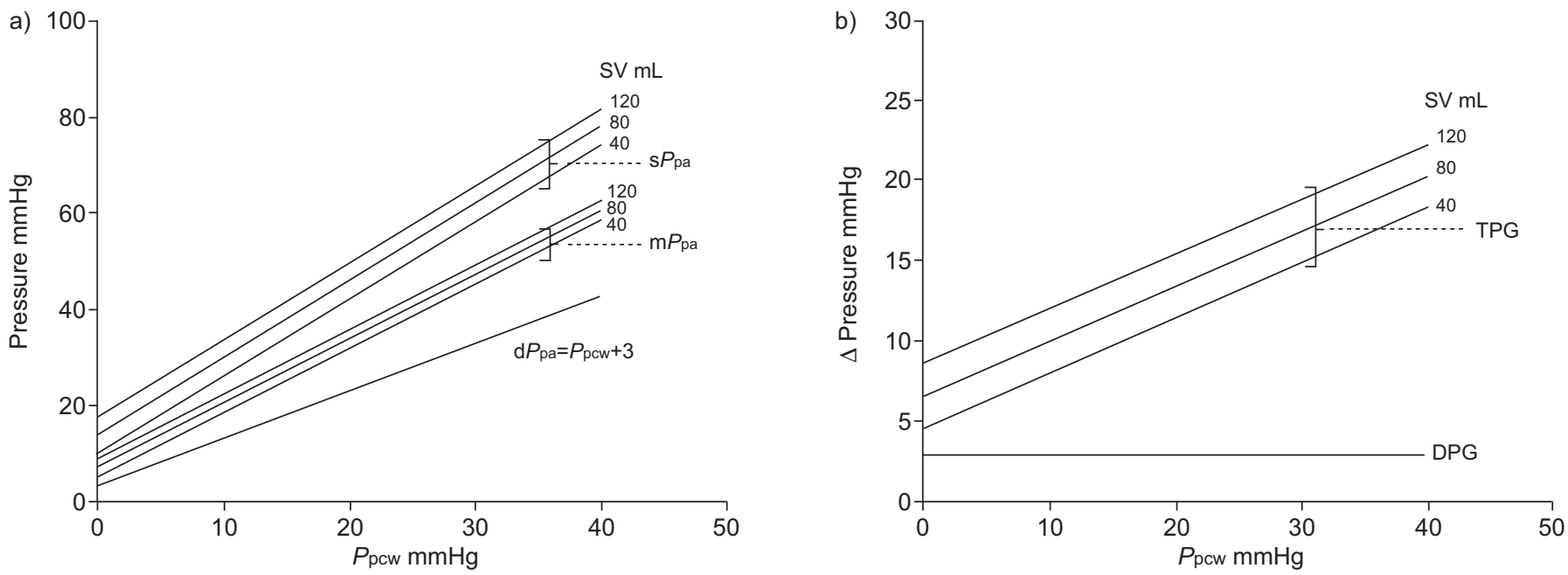

FIGURE 5. Effects of pulmonary capillary wedge pressure ( $P$ pcw) and stroke volume $(\mathrm{SV})$ on systolic $(\mathrm{s})$, diastolic $(\mathrm{d})$ and mean $(\mathrm{m})$ pulmonary arterial pressures $(P$ pa). If $P$ pcw is directly transmitted to diastolic $P$ pa, there is a disproportional increase in systolic $P$ pa and mean Ppa depending on SV. The transpulmonary pressure gradient (TPG) increases, but the diastolic $P$ pa/Ppcw gradient (DPG) is independent of both Ppcw and SV. The equations from [14] were used to model the response of the vasculature.

DPG below or above $5 \mathrm{mmHg}$, to discern passive upstream transmission of Pla from increased PVR due to pulmonary vasoconstriction and/or pulmonary vascular structural changes. The last step is cardiac output, being normal or decreased in heart failure, or normal or increased in hypervolaemia and/or increased venous return on low systemic vascular resistance in anaemia, systemic shunts, or sepsis. The arteriovenous oxygen content difference $\left(\mathrm{Dav}, \mathrm{O}_{2}\right)$ can be used as a surrogate of cardiac output, as the Fick equation predicts that both variables are inversely correlated at any given value of oxygen uptake [21]. The $\mathrm{Dav}, \mathrm{O}_{2}$ is a useful internal control to integrate in haemodynamic measurements at right heart catheterisation, as errors on a measurement of cardiac output are always possible. Low systemic blood pressure argues in favour of a septic complication. Cut-off values for cardiac output or blood pressure are not

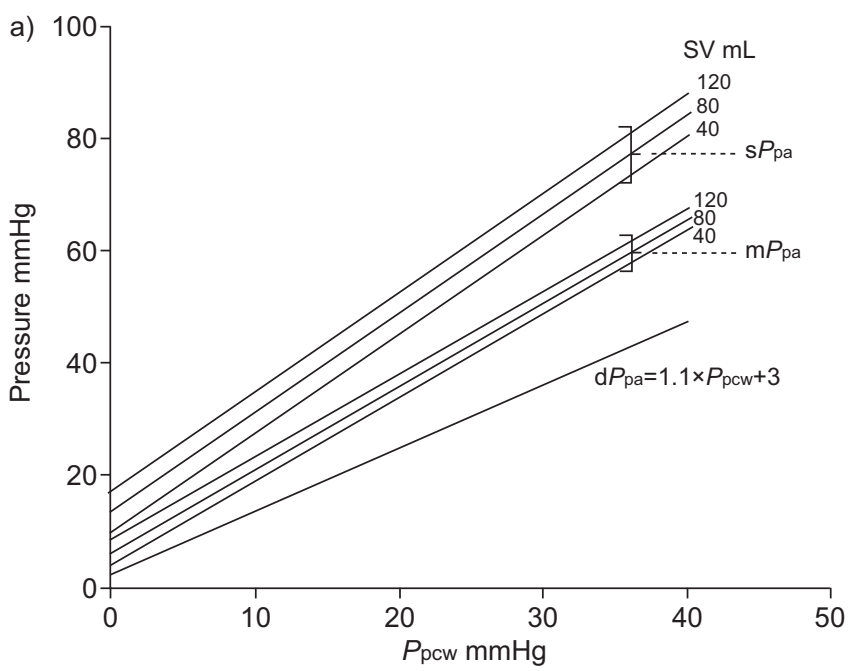

proposed because of insufficient evidence and the desire to propose a decision tree with sufficient flexibility to assist, rather than impose, clinical decisions.

\section{CONCLUSION AND PERSPECTIVE}

In 1971, HARVEY et al. [14] stated: "The pulmonary circulation has been under intensive study by innumerable investigators for almost 30 years. From the vast amounts of carefully accumulated data, certain conclusions can be drawn". More than 40 yrs later, there has been progress, but also some persisting misconceptions. A typical example is the reliance of the TPG for the differential diagnosis of pulmonary vascular disease. The present discussion shows how the TPG may over-diagnose or underdiagnose pulmonary vascular disease in left heart conditions associated with an increased pulmonary venous pressure, and

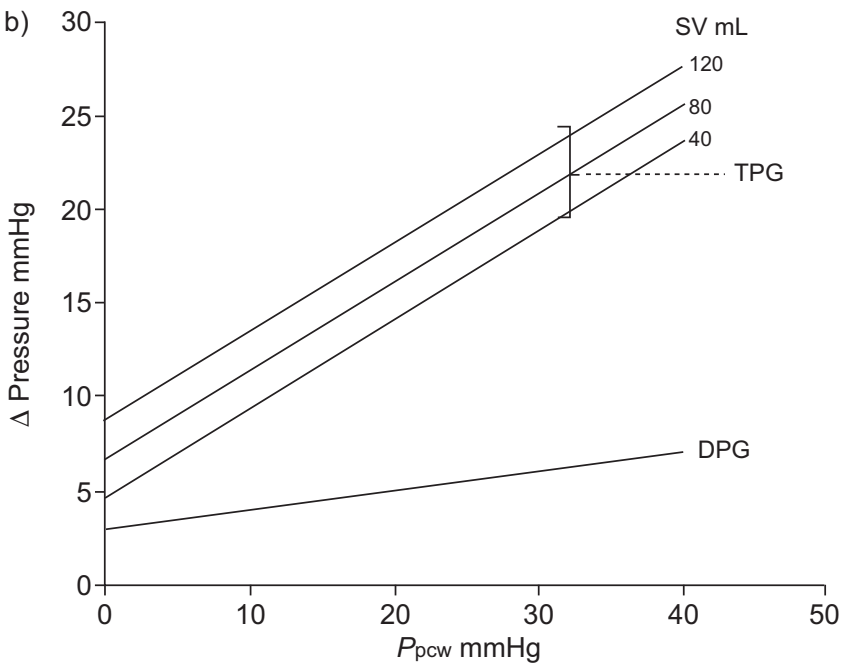

FIGURE 6. Effects of pulmonary capillary wedge pressure ( $P$ pcw) and stroke volume (SV) on systolic (s), diastolic (d) and mean (m) pulmonary arterial pressures ( $P$ pa). If diastolic $P$ pa increases more than $P$ pcw, there is an out of proportion increase in systolic $P$ pa and mean $P$ pa that is a function of SV. The transpulmonary pressure gradient (TPG) increases. The diastolic $P$ pa/Ppcw gradient (DPG) increases linearly, but only slightly, with $P$ pcw and is independent of SV. The equations from [14] were used to model the response of the vasculature. 


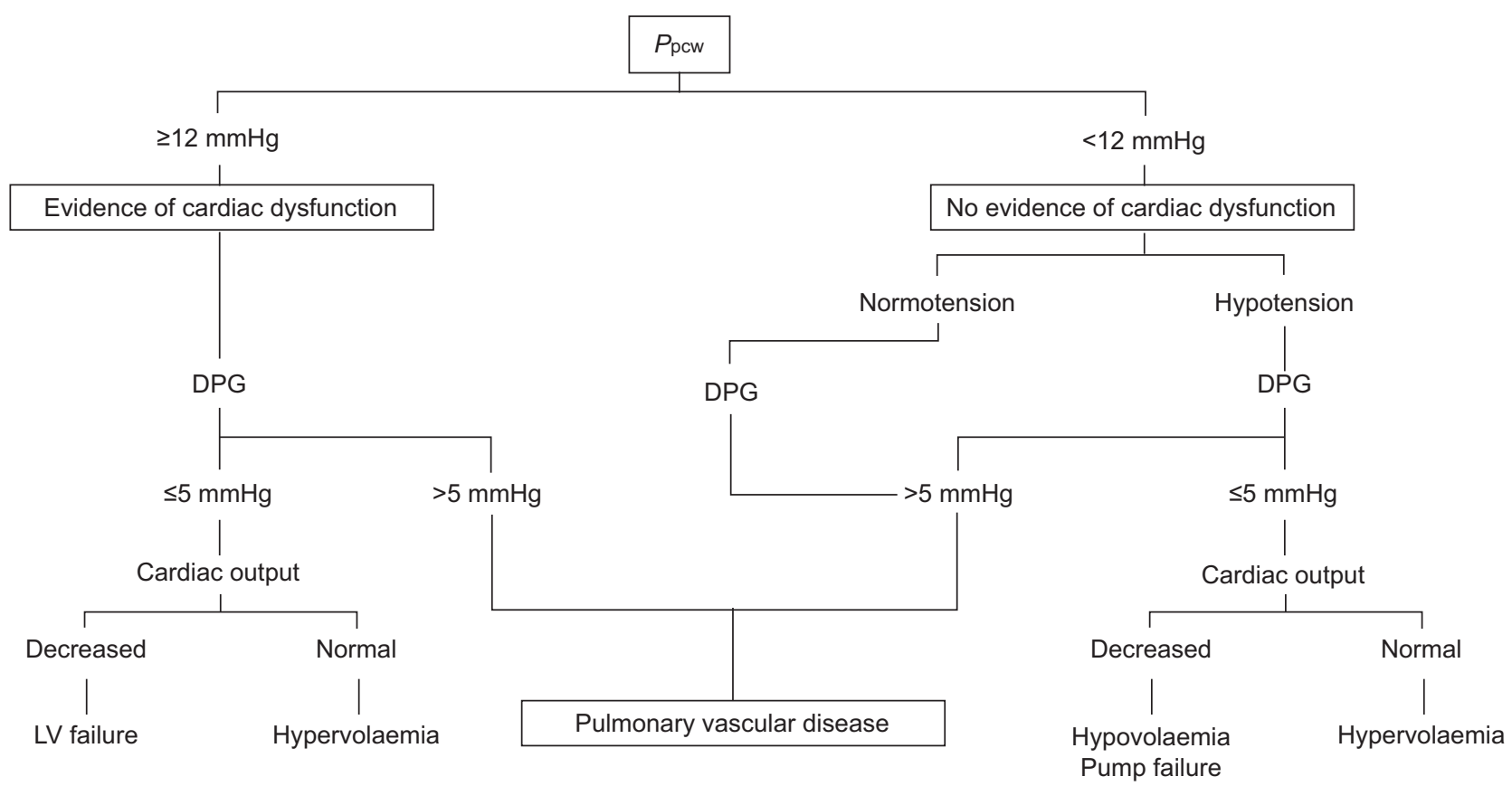

FIGURE 7. Pulmonary capillary wedge ( $P$ pcw)-derived algorithm for the diagnosis of heart failure (low or high output) versus intrinsic pulmonary vascular disease. A cutoff value of $12 \mathrm{mmHg}$ is selected as a true upper limit for normal $P$ pcw measured at right heart catheterisation. If the cardiac catheterisation is left, $P$ pcw is replaced by left ventricular (LV) end-diastolic pressure, with a cut-off value of $15 \mathrm{mmHg}$. Systemic hypotension is considered to make the decision tree applicable to septic shock. DPG diastolic pulmonary arterial pressure/Ppow gradient.

that this is, to a large extent, avoided by the use of a DPG. The DPG combined with clinical probability assessment, absolute values of $P$ pcw or $P$ la, cardiac output or $\mathrm{DavO}_{2}$ and blood pressure measurements appear to be more useful for the diagnosis of "out of proportion" pulmonary hypertension secondary to left heart conditions, and may help in the management of critically ill patients with sepsis or acute lung injury.

It is of course understood that the presently proposed diagnostic tree requires prospective validation. This could be undertaken along with TPG-based algorithms as all these measurements are currently performed during diagnostic catheterisations.

As a final word of caution, one should never forget that decision trees based on single measurement cut-off values are vulnerable. This was recently illustrated by a poor agreement between right and left heart catheterisation measurements of $P$ pcw and $P$ la in a large patient population [23], and persistant discussion on how optimally measure Ppcw [24]. The measurement of diastolic $P$ pa is more exposed than mean $P$ pa to motion artefacts and inadequate dynamic responses due to over damping or under damping (insufficient or excessive flushing of the manometer fluid-filled catheter system). This may explain why Ppcw higher than diastolic P pa are sometimes observed [14], although this is physically impossible. Furthermore, diagnostic cut-off values do not necessarily coincide with upper limits of normal [1,2], and some uncertainty also remains with the limits of normal of pulmonary haemodynamics in respect of aging, as the number of reported studies in old but healthy subjects remains limited [10-12, 14]. Thus the upper limit of normal for the DPG may have to be increased in elderly, healthy subjects. Great care must be applied in the quality control of measurements and they should be cross-checked with clinical probability assessments and alternative imaging techniques should be pursued, whenever possible.

\section{STATEMENT OF INTEREST}

A statement of interest for R. Naeije can be found at www.erj. ersjournals.com/site/misc/statements.xhtml

\section{REFERENCES}

1 Galiè N, Hoeper M, Humbert M, et al. Guidelines for the diagnosis and treatment of pulmonary hypertension. The Task Force for the Diagnosis and Treatment of Pulmonary Hypertension of the European Society of Cardiology (ESC) and the European Respiratory Society (ERS), endorsed by the International Society of Heart and Lung Transplantation (ISHLT). Eur Respir J 2009; 34: 1219-1263.

2 McLaughlin VV, Archer SL, Badesch DB, et al. ACCF/AHA 2009 expert consensus document on pulmonary hypertension: a report of the American College of Cardiology Foundation Task Force on Expert Consensus Documents and the American Heart Association developed in collaboration with the American College of Chest Physicians, American Thoracic Society, Inc. and the Pulmonary Hypertension Association. Circulation 2009; 119: 2250-2294

3 Naeije R, Lipski A, Abramowicz M, et al. Nature of pulmonary hypertension in congestive heart failure. Effects of cardiac transplantation. Am J Respir Crit Care Med 1994; 147: 881-887.

4 Naeije R. Pulmonary vascular resistance: a meaningless variable? Intensive Care Med 2003; 29: 526-529.

5 Permutt S, Bromberger-Barnea B, Bane HN. Alveolar pressure, pulmonary venous pressure and the vascular waterfall. Med Thorac 1962; 19: 239-260. 
6 West JB, Dollery CT, Naimark A. Distribution of blood flow in isolated lung: relation to vascular and alveolar pressures. J Appl Physiol 1964; 19: 713-724.

7 Zapol WM, Snider MT. Pulmonary hypertension in severe acute respiratory failure. N Engl J Med 1977; 296: 476-480.

8 Leeman M, Lejeune P, Closset J, et al. Nature of pulmonary hypertension in canine oleic acid pulmonary edema. J Appl Physiol 1990; 69: 293-298.

9 Linehan JH, Haworth ST, Nelin LD, et al. A simple distensible model for interpreting pulmonary vascular pressure-flow curves. J Appl Physiol 1992; 73: 987-994.

10 Reeves JT, Linehan JH, Stenmark KR. Distensibility of the normal human lung circulation during exercise. Am J Physiol Lung Cell Mol Physiol 2005; 288: L419-L425.

11 Argiento $\mathrm{P}$, Chesler N, Mulè M, et al. Exercise stress echocardiography for the study of the pulmonary circulation. Eur Respir $J$ 2010; 35: 1273-1278.

12 Argiento P, Vanderpool RR, Mule M, et al. Exercise stress echocardiography of the pulmonary circulkation: limits of normal and gender differences. Chest 2012 [Epub ahead of print DOI:10.1378/chest.12-0071].

13 Groepenhoff H, Overbeek MJ, Mulè M, et al. Exercise pathophysiology in patients with chronic mountain sickness. Chest 2012 [Epub ahead of print DOI:10.1378/chest.11-2845].

14 Harvey RM, Enson Y, Ferrer MI. A reconsideration of the origins of pulmonary hypertension. Chest 1971; 59: 82-94.

15 Chemla D, Castelain V, Humbert M, et al. New formula for predicting mean pulmonary artery pressure using systolic pulmonary artery pressure. Chest 2004; 126: 1313-1317.

16 Syyed R, Reeves JT, Welsh D, et al. The relationship between the components of pulmonary artery pressure remains constant under all conditions in both health and disease. Chest 2008; 133 633-639.

17 Chemla D, Castelain V, Provencher S, et al. Evaluation of various empirical formulas for estimating mean pulmonary artery pressure by using systolic pulmonary artery pressure in adults. Chest 2009; 135: 760-768.

18 Lankhaar JW, Westerhof N, Faes TJ, et al. Quantification of right ventricular afterload in patients with and without pulmonary hypertension. Am J Physiol Heart Circ Physiol 2006; 291: H1731-H1737.

19 Saouti N, Westerhof N, Helderman F, et al. Right ventricular oscillatory power is a constant fraction of total power irrespective of pulmonary artery pressure. Am J Respir Crit Care Med 2010; 182: 1315-1320.

20 Tedford RJ, Hassoun PM, Mathai SC, et al. Pulmonary capillary wedge pressure augments right ventricular pulsatile loading. Circulation 2012; 125: 289-297.

21 Stevens PM. Assessment of acute respiratory failure: cardiac versus pulmonary causes. Chest 1975; 67: 1-2.

22 Paulus WJ, Tschöpe C, Sanderson JE, et al. How to diagnose diastolic heart failure: a consensus statement on the diagnosis of heart failure with normal left ventricular ejection fraction by the Heart Failure and Echocardiography Associations of the European Society of Cardiology. Eur Heart J 2007; 28: 2539-2550.

23 Halpern SD, Taichman DB. Misclassification of pulmonary hypertension due to reliance on pulmonary capillary wedge pressure rather than left ventricular end-diastolic pressure. Chest 2009; 136: 37-43.

24 Ryan JJ, Rich JD, Thiruvoipati $\mathrm{T}$, et al. Current practice for determining pulmonary capillary wedge pressure predisposes to serious errors in the classification of patients with pulmonary hypertension. Am Heart J 2012; 163: 589-594. 